\title{
INTERPRETATION OF THE POLARIZATION MEASUREMENTS OF THE DOUBLE GLUSTER IN PERSEUS
}

K. SERKOWSK I

Astronomical Observatory of the University of Warsaw, Poland

The stars in the Double Gluster in Perseus whose angular distance is small have similar polarization and similar colour excesses. Quantitative description of this effect is the purpose of this paper.

The observational data are taken from polarization measurements performed by Hiltner [1] and blue-yellow colour excesses obtained from data by Johnson and Morgan [2] and by Johnson and Hiltner [3]. The above investigations seem to be the most extensive ones; the measurements by other authors were not included in order not to destroy the uniformity of data.

The polarization is most conveniently described by the parameters $Q, U$, proportional to the Stokes parameters and defined by

$$
\begin{aligned}
& Q=p \cos 2(\theta-\bar{\theta}), \\
& U=p \sin 2(\theta-\bar{\theta}),
\end{aligned}
$$

where $p$ is the amount of polarization (in magnitudes) and $\theta$ is the position angle of the electric vector. For the cluster stars the mean square deviation of the position angle $\theta$ from the mean value $\bar{\theta}$ is only $\pm 4^{\circ}$ and therefore $Q \simeq p$.

Within a radius of $2^{\circ}$ from the centre of the Double Cluster the polarization of ninety-two stars was measured by Hiltner. These stars were connected in pairs in all possible combinations. The pairs were distributed in groups according to the angular distance $\phi$ of the stars in pairs. For every group the mean square of the difference in the parameters $Q$ and $U$ was computed for the stars in pairs. The values obtained in this way (given in Fig. I and Fig. 2) show that the mean square of the difference in every Stokes parameter for the close pairs is half that for the distant ones.

The mean squares of the differences in the colour excess $E$ show still more 


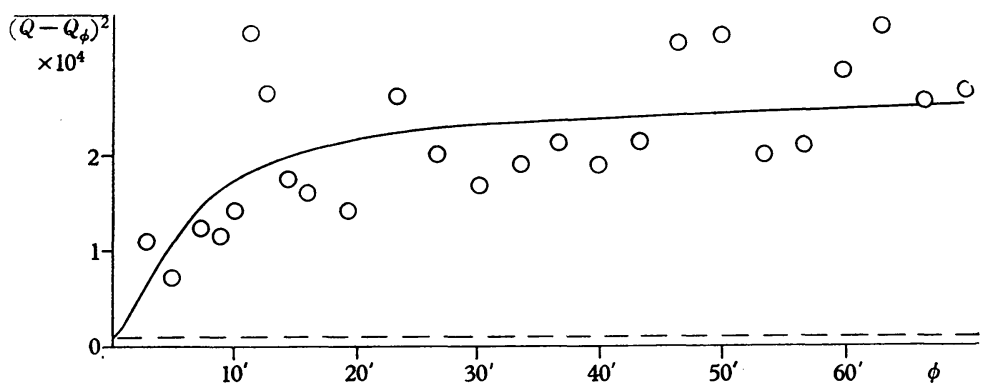

Fig. 1. The mean squares of differences in the Stokes parameter $Q$ (amount of polarization) versus angular distance of the stars. The dashed line represents the errors of measurements, the solid line was computed from formula (8) for the micro-scale $s_{o q}=\mathrm{I} \cdot 5 \mathrm{pc}$.

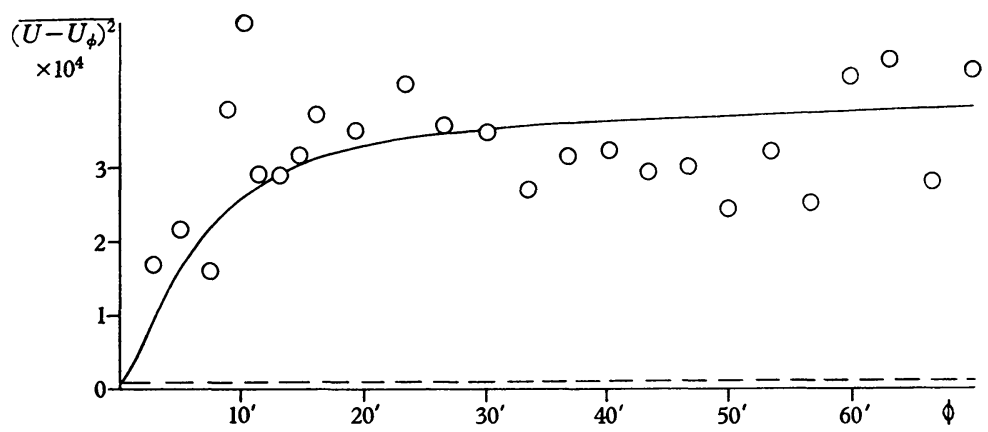

Fig. 2. The mean squares of differences in the Stokes parameter $U$.

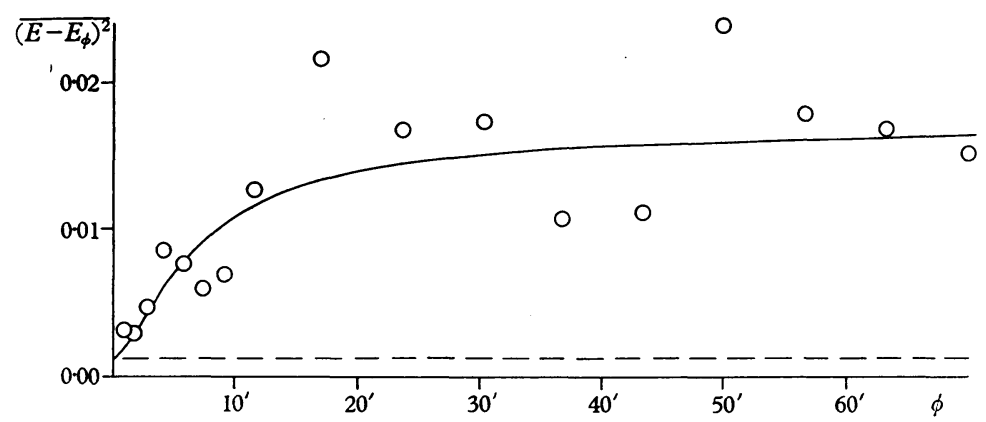

Fig. 3. The mean squares of differences in the colour excess. The solid line was computed for the micro-scale $s_{o \delta}=\mathrm{I} \cdot 8 \mathrm{pc}$.

distinctly the dependence on the angular distance of the stars forming the pair (Fig. 3).

Let $H_{x}, H_{y}$ and $H_{z}$ be the components of the galactic magnetic field in the co-ordinate system in which $O Y$ is in the direction of the light ray and $O Z$ is perpendicular to the galactic plane. It is assumed after Davis [4] that 
the increase of the parameters $Q, U$, over the path $d s$ is

$$
\begin{gathered}
d Q=c \rho\left(H_{x}^{2}-H_{z}^{2}\right) d s, \\
d U=2 c \rho H_{x} H_{z} d s,
\end{gathered}
$$

where $\rho$ is the density of absorbing matter and $c=$ const. The similar increase of the colour excess $E$ can be given by

$$
d E=c^{\prime} \rho d s \quad c^{\prime}=\text { const. }
$$

Applying the method proposed by Chandrasekhar and Münch [5] the random functions $\delta(s), q(s), u(s)$, are defined by

$$
\begin{gathered}
\rho=\bar{\rho}[\mathrm{I}+\delta(s)], \\
\rho\left(H_{x}^{2}-H_{z}^{2}\right)=\bar{\rho}\left(\overline{H_{x}^{2}}-\overline{H_{z}^{2}}\right)[\mathrm{I}+q(s)], \\
2 \rho H_{x} H_{z}=\bar{\rho}\left(\overline{H_{x}^{2}}-\overline{H_{z}^{2}}\right) u(s),
\end{gathered}
$$

where $s$ is a radius vector of given point in space. The assumption is made that $\delta(s), q(s), u(s)$, can be treated as homogeneous stochastical processes fulfilling the relations

$$
\overline{\delta(s)}=\overline{q(s)}=\overline{u(s)}=0,
$$

$$
\begin{array}{cc}
\overline{\delta^{2}(s)}=\alpha^{2}=\mathrm{const}, \quad \overline{q^{2}(s)}=\beta^{2}=\mathrm{const}, \quad \overline{u^{2}(s)}=\gamma^{2}=\mathrm{const}, \\
\overline{\delta_{1} \delta_{2}}=\alpha^{2} R\left(\left|s_{1}-s_{2}\right|\right), \quad \overline{q_{1} q_{2}}=\beta^{2} R\left(\left|s_{1}-s_{2}\right|\right), \quad \overline{u_{1} u_{2}}=\gamma^{2} R\left(\left|s_{1}-s_{2}\right|\right),
\end{array}
$$

where $\left|s_{1}-s_{2}\right|$ is distance between the points in which the values of the functions are respectively $\delta_{1}$ and $\delta_{2}$, etc., and $R\left(\left|s_{1}-s_{2}\right|\right)$ are the correlation functions which following Chandrasekhar and Münch are specified as

$$
R\left(\left|s_{1}-s_{2}\right|\right)=\exp \left(-\left|s_{1}-s_{2}\right| / s_{o k}\right),
$$

where instead of $k$ should be put respectively indices $\delta, q, u$.

From the preceding formulae by numerical integration was obtained

$$
\begin{aligned}
& \overline{(E-\bar{E})^{2}}=2 \bar{E}^{2} \alpha^{2} s_{o \delta} / S, \\
& \overline{(Q-\bar{Q})^{2}}=2 \bar{Q}^{2} \beta^{2} s_{o q} / S, \\
& \overline{U^{2}}=2 \bar{Q}^{2} \gamma^{2} s_{\text {oul }} / S, \\
& \overline{\left(E-E_{\phi}\right)^{2}} \simeq 2 \overline{(E-\bar{E})^{2}}\left[\mathrm{I}-\exp \left(-a \phi S / s_{o \delta}\right)\right], \\
& \overline{\left(Q-Q_{\phi}\right)^{2}} \simeq 2 \overline{(Q-\bar{Q})^{2}}\left[\mathrm{I}-\exp \left(-a \phi S / s_{o q}\right)\right] \text {, } \\
& \overline{\left(U-U_{\phi}\right)^{2}} \simeq 2 \overline{U^{2}}\left[\mathrm{I}-\exp \left(-a \phi S / s_{\text {ou }}\right)\right],
\end{aligned}
$$

where $a=0.23$ is constant resulting from the integration and $S=2300 \mathrm{pc}$ is the distance of Double Gluster. 
After eliminating the dependence of $E$ and $p$ on the galactic latitude (assumed to be linear) the observational data used in the present paper give

$$
\begin{aligned}
\bar{E} & =\mathrm{O}^{\mathrm{m}} \cdot 58, \quad \bar{Q}=\mathrm{O}^{\mathrm{m}} \cdot \mathrm{O} 79, \\
\overline{(E-\bar{E})^{2}}=9 \cdot 10^{-3}, \quad \overline{(Q-\bar{Q})^{2}} & =\mathrm{I} \cdot 3 \cdot 10^{-4}, \quad \overline{U^{2}}=2 \cdot 0 \cdot 10^{-4} .
\end{aligned}
$$

All the three parameters $E, p, U$, are uncorrelated. Obviously, $E$ and $p$ are uncorrelated only when we are examining the stars in the narrow interval of the galactic latitude (e.g. $\mathrm{I}^{\circ}$ ) since both $E$ and $p$ are dependent on the galactic latitude. Further, the correlation between $p$ and the absolute magnitude of the stars, suggested by Dombrowski [6] was not confirmed.

Applying the formula (7) to data plotted on Fig. 3 the micro-scale of the fluctuations in the density of absorbing matter $s_{o \delta}=\mathrm{r} \cdot 8 \mathrm{pc}$ is obtained. The data given in Fig. I and Fig. 2 can be represented by the formulae (8), (9), with only one value of the micro-scale $s_{o q}=s_{o u}=\mathrm{I} \cdot 5 \mathrm{pc}$.

Formulae (4)-(6) when combined with the foregoing values give

$$
\begin{aligned}
& \alpha^{2}=\overline{(\rho-\bar{\rho})^{2}} / \bar{\rho}^{2}=15, \\
& \beta^{2}=\mathrm{I} 6, \\
& \gamma^{2}=25 .
\end{aligned}
$$

Let us consider the large-scale homogeneous magnetic field on which are imposed fluctuations perpendicular to the mean direction of the field. In the region of the Double Cluster the mean direction of the magnetic field coincides approximately with the $X$-axis of the co-ordinate system formerly introduced. So it can be assumed that the fluctuations are isotropic in the $Y Z$-plane and in the direction of the $X$-axis they disappear. In this case from the equations $(I)-(3)$ we obtain

$$
\begin{aligned}
& \beta^{2}=\alpha^{2}+2\left(\mathrm{I}+\alpha^{2}\right){\overline{H_{z}^{2}}}_{z}^{2} /\left(\bar{H}_{x}^{2}-\overline{H_{z}^{2}}\right)^{2}, \\
& \gamma^{2}=4\left(\mathrm{I}+\alpha^{2}\right){\overline{H_{x}}}_{x}^{2} \overline{H_{z}^{2}} /\left({\overline{H_{x}}}_{x}^{2}-{\overline{H_{z}^{2}}}^{2}\right)^{2} .
\end{aligned}
$$

Substituting here the values (I I), (I 2) found from the polarization data we obtain $\alpha^{2}=$ I 3 which agrees sufficiently well with the value (10) resulting from the analysis of the colour excesses. In this way we obtain also the root-mean-square angular deviation of the lines of force from a uniform direction

$$
\alpha_{1}=\left(2 \overline{H_{z}^{2}} / \bar{H}_{x}^{2}\right)^{1 / 2}=0 \cdot 7 \mathrm{I}
$$

about three times greater than the result obtained by Davis [4]. 


\section{REFERENCES}

[1] Hiltner, W. A. Astrophys. J. 114, 24I, 195I and Astrophys. J., 120, 454, 1954.

[2] Johnson, H. L. and Morgan, W. W. Astrophys. J. 122, 429, 1955.

[3] Johnson, H. L. and Hiltner, W. A. Astrophys. J. 123, 267, 1956.

[4] Davis, L. Vistas in Astronomy, vol. I, ed. by A. Beer, p. 336, (Pergamon Press, London, 1955).

[5] Chandrasekhar, S. and Münch, G. Astrophys. J. 115, I03, 1952.

[6] Dombrowski, W. A. Russ. Astr. J. 3o, 6o3, r 953.

\section{Discussion}

Spitzer: How great are the probable errors of the obtained values of the micro-scale?

Serkowski: I think that the probable errors of $s_{o \delta}$ and $s_{o q}$ are $30 \%$ or somewhat more. The value of $s_{o u}$ is much more inaccurate. 